APPLICATIONES MATHEMATICAE

26,1 (1999), pp. 63-83

D. HAMADOUCHE and C. SUQUET (Lille)

\title{
WEAK HÖLDER CONVERGENCE OF PROCESSES WITH APPLICATION TO THE PERTURBED EMPIRICAL PROCESS
}

Abstract. We consider stochastic processes as random elements in some spaces of Hölder functions vanishing at infinity. The corresponding scale of spaces $C_{0}^{\alpha, 0}$ is shown to be isomorphic to some scale of Banach sequence spaces. This enables us to obtain some tightness criterion in these spaces. As an application, we prove the weak Hölder convergence of the convolutionsmoothed empirical process of an i.i.d. sample $\left(X_{1}, \ldots, X_{n}\right)$ under a natural assumption about the regularity of the marginal distribution function $F$ of the sample. In particular, when $F$ is Lipschitz, the best possible bound $\alpha<1 / 2$ for the weak $\alpha$-Hölder convergence of such processes is achieved.

1. Introduction. The weak convergence of a sequence $\left(\xi_{n}, n \geq 1\right)$ of stochastic processes is classically studied in the Skorokhod spaces for processes having jumps or else in some space $C(T)$ of continuous functions. In many usual cases, the paths of $\xi_{n}$ and of the limiting process $\xi$ exhibit more regularity than the bare continuity. For instance, the Donsker-Prokhorov invariance principle establishes the $C(0,1)$-weak convergence to the Brownian motion $W$ of the random polygonal lines $\xi_{n}$ interpolating the (centered and normalized) partial sums of an i.i.d. sequence. Here the paths of $W$ are (with probability one) of Hölder regularity $\alpha$ for any $\alpha<1 / 2$ and those of $\xi_{n}$ are of Hölder regularity 1. This remark was exploited by Lamperti [11] to prove the same invariance principle in the space $\mathrm{H}_{\alpha}(0,1)$ of $\alpha$-Hölder continuous functions for any $\alpha<1 / 2$. As pointed out by Lamperti, this is an improvement on the $C(0,1)$-invariance principle since $\mathrm{H}_{\alpha}(0,1)$ is topologically imbedded in $C(0,1)$. Roughly speaking, if $\mathcal{X}$ is some space of functions

1991 Mathematics Subject Classification: 60B10, 60F05, 62G30.

Key words and phrases: Brownian bridge, Hölder space, perturbed empirical process, Schauder decomposition, tightness, triangular functions. 
on $T$, embedded in $C(T)$, there are more continuous functionals on $\mathcal{X}$ than on $C(T)$, so $\mathcal{X}$-weak convergence is a stronger result than $C(T)$ convergence. Clearly, when $\mathcal{X}$ belongs to some scale of function spaces, the best result which can be expected in this direction is the weak convergence in the space of the scale whose elements have the best common regularity shared by the paths of $\xi_{n}$ and $\xi$.

Unfortunately, this is not always possible. In [8], Hamadouche considers the (centered, normalized) frequencies polygon of a sample $\left(U_{1}, \ldots, U_{n}\right)$ of independent uniformly $[0,1]$-distributed random variables. He proves the weak convergence of $\xi_{n}$ to the Brownian bridge $B$ in the space $\mathrm{H}_{\alpha}(0,1)$ of $\alpha$-Hölder functions for any $\alpha<1 / 4$. This bound is shown to be optimal. Still, the Brownian bridge has (with probability one) $\alpha$-Hölderian paths for any $\alpha<1 / 2$. Another smoothing procedure based on convolution kernels is proposed in [8] for the uniform empirical process and allows reaching the expected bound $\alpha<1 / 2$ for the weak Hölder convergence to the Brownian bridge.

Nevertheless, from the statistical point of view, it should be noticed that the smoothing by convolution of the usual empirical process does not commute with the change of variable $U_{i}=F\left(X_{i}\right)$ where $F$ is the marginal distribution function of an i.i.d. sample $\left(X_{1}, \ldots, X_{n}\right)$. So the last result, on weak Hölder convergence for any $\alpha<1 / 2$, cannot be applied to the smoothed empirical process of $\left(X_{1}, \ldots, X_{n}\right)$ and the Hölder weak convergence of this process should be studied directly.

The aim of this paper is to provide a rather general framework for the study of Hölder weak convergence of processes indexed by the real line. To this end we introduce a scale of spaces $C_{0}^{\alpha}$ of functions with global Hölder regularity of order $\alpha$ and vanishing at infinity. For separability reasons we also consider the subspaces $C_{0}^{\alpha, 0}$ (precise definitions are given in Section 2). All these spaces are analysed by triangular Schauder functions and shown to be isomorphic to some Banach sequence spaces. Using a general result of Suquet [16], we give in Section 3 several conditions for the $C_{0}^{\alpha, 0}$ tightness of a sequence of stochastic processes. As an application we treat in Section 4 the problem of weak Hölder convergence for the convolution smoothed empirical process $\xi_{n}$. In particular, when the marginal distribution function $F$ of the sample is smooth enough, $\xi_{n}$ converges weakly in $C_{0}^{\alpha, 0}(\mathbb{R})$ to a centered Gaussian process $\xi$ with covariance $F(x \wedge y)-F(x) F(y)$ for any $\alpha<1 / 2$.

This method of studying stochastic processes via sequence spaces goes back at least to Ciesielski [3], [4]. The analytical part of our results is an extension of Ciesielski's results [3] about the spaces $\mathrm{H}_{\alpha}(0,1)$. Recent developments involving this method can be found in the paper [5] by Ciesielski, Kerkyacharian and Roynette. 
2. The functional framework. For any $0<\alpha<1$, define $C_{0}^{\alpha}(\mathbb{R})$ as the space of functions $f$ such that

$$
\begin{gathered}
\lim _{|x| \rightarrow \infty} f(x)=0 \quad \text { and } \\
\|f\|_{\alpha}:=\|f\|_{\infty}+w_{\alpha}(f, 1)<\infty,
\end{gathered}
$$

where $\|f\|_{\infty}:=\sup \{|f(x)|: x \in \mathbb{R}\}$ and, for $\delta>0$,

$$
w_{\alpha}(f, \delta):=\sup _{\substack{-\infty<s, t<\infty \\ 0<|t-s| \leq \delta}} \frac{|f(t)-f(s)|}{|t-s|^{\alpha}} .
$$

Remark. The functional $w_{\alpha}(f, 1)$ is a priori only a seminorm. But here $f$ vanishes at infinity, so $w_{\alpha}(f, 1)=0$ if and only if $f=0$ and $w_{\alpha}(f, 1)$ is a norm on $C_{0}^{\alpha}$. The presence of the extra term $\|f\|_{\infty}$ in (2) should look rather artificial at first sight. In fact, the situation is quite different from the Hölder space $\mathrm{H}_{\alpha}(0,1)$ studied by Ciesielski and this extra term is really useful, as shown by the following example. Consider the triangular function $f_{n}$ with nodes at the points $(0,0),(n, \sqrt{n})$ and $(2 n, 0)$. We have $w_{\alpha}\left(f_{n}, 1\right)=n^{-1 / 2}$ while $\|f\|_{\infty}=n^{1 / 2}$, hence the two norms $\|f\|_{\alpha}$ and $w_{\alpha}(f, 1)$ are not equivalent. Moreover this shows that the convergence in $C_{0}^{\alpha}$ endowed with the norm $w_{\alpha}(\cdot, 1)$ does not imply even pointwise convergence.

It is easily verified that $C_{0}^{\alpha}(\mathbb{R})$ equipped with the norm \|\|$_{\alpha}$ is a Banach space. Its analysis by some triangular Schauder functions to be developed below will show that $C_{0}^{\alpha}(\mathbb{R})$ contains a subspace isomorphic to $\ell^{\infty}(\mathbb{N})$ and hence is not separable. To remedy this drawback, we introduce the closed subspace $C_{0}^{\alpha, 0}(\mathbb{R})$ defined by

(4) $\quad f \in C_{0}^{\alpha, 0}(\mathbb{R})$ if and only if $f \in C_{0}^{\alpha}(\mathbb{R})$ and $\lim _{\delta \rightarrow 0} w_{\alpha}(f, \delta)=0$.

For simplicity, from now on we abbreviate $C_{0}^{\alpha}(\mathbb{R})$ and $C_{0}^{\alpha, 0}(\mathbb{R})$ to $C_{0}^{\alpha}$ and $C_{0}^{\alpha, 0}$.

For any $f \in C_{0}^{\alpha}$ and each $j \in \mathbb{N}$, let $E_{j} f$ be the polygonal line interpolating $f$ at the points of abscissas $r_{j, k}=k 2^{-j}, k \in \mathbb{Z}$. Elementary computations give the estimates

$$
\begin{aligned}
\left\|E_{j} f-f\right\|_{\infty} & \leq 2^{1-j \alpha} w_{\alpha}\left(f, 2^{-j}\right), \\
w_{\alpha}\left(E_{j} f-f, 1\right) & \leq 4 w_{\alpha}\left(f, 2^{-j}\right),
\end{aligned}
$$

from which the separability of $C_{0}^{\alpha, 0}$ easily follows.

We now prove that $C_{0}^{\alpha, 0}$ is Schauder decomposable, that is, there is some sequence $\left(\mathcal{X}_{i}: i \in \mathbb{N}\right)$ of closed subspaces such that

$$
C_{0}^{\alpha, 0}=\bigoplus_{i \in \mathbb{N}} \mathcal{X}_{i}
$$


where the direct sum is topological (i.e. the canonical projections on the $\mathcal{X}_{i}$ 's are continuous). For the general theory of Schauder decompositions, we refer to Singer [14]. This decomposition is useful to obtain the isomorphism between $C_{0}^{\alpha, 0}$ and some Banach sequence space and to study the tightness of processes with paths in $C_{0}^{\alpha, 0}$ by the method of [16].

The proof is based on the analysis of $C_{0}^{\alpha, 0}$ by two scales of triangular functions constructed as follows. First define

$$
\Delta^{*}(t)= \begin{cases}1+t & \text { if }-1 \leq t \leq 0 \\ 1-t & \text { if } 0 \leq t \leq 1 \\ 0 & \text { elsewhere }\end{cases}
$$

and

$$
\Delta_{k}^{*}(t)=\Delta^{*}(t-k), \quad t \in \mathbb{R}, k \in \mathbb{Z} .
$$

The second scale is the classical Faber-Schauder one obtained by translations and dyadic changes of scales from the triangular function

$$
\Delta(t)= \begin{cases}2 t & \text { if } 0 \leq t \leq 1 / 2 \\ 2(1-t) & \text { if } 1 / 2 \leq t \leq 1 \\ 0 & \text { elsewhere }\end{cases}
$$

putting

$$
\Delta_{j, k}(t)=\Delta\left(2^{j} t-k\right), \quad t \in \mathbb{R}, j \in \mathbb{N}, k \in \mathbb{Z} .
$$

To explain the respective roles played by these two scales, let us recall the Faber-Schauder algorithm of decomposition of the space $C(0,1)$. If $f$ is a continuous function on $[0,1]$ with $f(0)=f(1)=0$, its projection $P_{1} f$ on (the vector line spanned by) $\Delta$ is simply the linear interpolation of $f$ between the points $0,1 / 2$ and 1 . Next, $f-P_{1} f$ vanishes at these points and the projection $P_{2} f$ of $f$ on the space spanned by $\Delta_{1,0}, \Delta_{1,1}$ is the linear interpolation of $f-P_{1} f$ at the points $0,1 / 4,1 / 2,3 / 4,1$, and so on. The initialization of the algorithm for general elements $f$ of $C(0,1)$ (not necessarily vanishing at 0 and 1) requires introducing to the scale $\left\{\Delta_{j, k}: j \in \mathbb{N}, 0 \leq k<2^{j}\right\}$ two extra functions $h_{-1}(x)=1$ and $h_{0}(x)=x$ such that $f-P_{0} f$ vanishes at 0 and 1, where $P_{0}$ is the projection given by $P_{0} f=f(0) h_{-1}+(f(1)-f(0)) h_{0}$. Geometrically speaking, the initialization step consists in subtracting the segment interpolating $f$ at the boundary points 0 and 1 .

This method cannot be applied directly to $C_{0}^{\alpha, 0}(\mathbb{R})$, since the boundary points are moved to infinity. The idea is then to subtract the polygonal line $E_{0} f$ interpolating $f$ at the integers. This is precisely the work assigned to the scale $\left\{\Delta_{k}^{*}: k \in \mathbb{Z}\right\}$, since the projection of $f \in C_{0}^{\alpha, 0}$ on the subspace generated by this scale is $E_{0} f$. Next, $f-E_{0} f$ vanishes at each integer $k \in \mathbb{Z}$ and can be treated locally on each interval $[k, k+1]$ by the Faber-Schauder algorithm using the scale $\left\{\Delta_{j, k}: j \in \mathbb{N}, k \in \mathbb{Z}\right\}$. It should be noticed here 
that the scale $\left\{\Delta_{k}^{*}: k \in \mathbb{Z}\right\}$ does not coincide with $\left\{\Delta_{-1, k}: k \in \mathbb{Z}\right\}$. In particular, the supports of $\Delta_{k}^{*}$ and $\Delta_{k+1}^{*}$ overlap.

ThEOREM 1. The space $C_{0}^{\alpha, 0}$ has the Schauder decomposition

$$
C_{0}^{\alpha, 0}=V_{0} \oplus \bigoplus_{j \in \mathbb{N}} W_{j}
$$

where $V_{0}$ is the closed subspace of $C_{0}^{\alpha}$ spanned by $\left\{\Delta_{k}^{*}: k \in \mathbb{Z}\right\}$ and, for $j \geq 0, W_{j}$ is the closed subspace of $C_{0}^{\alpha}$ spanned by $\left\{\Delta_{j, k}: k \in \mathbb{Z}\right\}$. The projections $E_{0}$ on $V_{0}$ and $D_{j}$ on $W_{j}$ are given by:

$$
\begin{aligned}
& E_{0} f=\sum_{k \in \mathbb{Z}} f(k) \Delta_{k}^{*}, \\
& D_{j} f=\left(E_{j+1}-E_{j}\right) f=\sum_{k \in \mathbb{Z}} c_{j, k}(f) \Delta_{j, k}, \quad j \geq 0,
\end{aligned}
$$

where

$$
c_{j, k}(f)=f\left(\left(k+\frac{1}{2}\right) 2^{-j}\right)-\frac{1}{2}\left\{f\left(k 2^{-j}\right)+f\left((k+1) 2^{-j}\right)\right\} .
$$

The series (13) and (14) converge in the strong topology of $C_{0}^{\alpha}$.

The proof relies on the following lemma which shows that the sequence of triangular functions spanning $V_{0}$ or $W_{j}$ is in fact a Schauder basis of these spaces.

Lemma 2. (i) The function $g$ belongs to $V_{0}$ if and only if $g=\sum_{k \in \mathbb{Z}} a_{k} \Delta_{k}^{*}$ for some sequence $\left(a_{k}\right)$ such that $\lim _{|k| \rightarrow \infty} a_{k}=0$. This representation is unique, the $a_{k}$ 's are given by $a_{k}=g(k)$ and the convergence of the series holds in the strong topology of $C_{0}^{\alpha}$.

(ii) For $j \geq 0, g$ belongs to $W_{j}$ if and only if $g=\sum_{k \in \mathbb{Z}} a_{j, k} \Delta_{j, k}$ for some sequence $\left(a_{j, k}\right)$ such that $\lim _{|k| \rightarrow \infty} a_{j, k}=0$. This representation is unique, the $a_{j, k}$ 's are given by $a_{j, k}=g\left((k+1 / 2) 2^{-j}\right)$ and the convergence of the series holds in the strong topology of $C_{0}^{\alpha}$.

Proof. If $g \in V_{0}$, then $g$ is the uniform limit on $\mathbb{R}$ of some sequence of functions $g_{n}$ which are affine on each interval $[l, l+1](l \in \mathbb{Z})$. Hence $g$ is itself affine on such intervals. Observing that $\Delta_{k}^{*}(l)=\delta_{k, l}$ (Kronecker symbol), we obtain the decomposition

$$
g(x)=\sum_{k \in \mathbb{Z}} g(k) \Delta_{k}^{*}(x), \quad x \in \mathbb{R},
$$

with (at least) pointwise convergence. Due to the values of the $\Delta_{k}^{*}(l)$ 's, such a decomposition is obviously unique. 

series

Conversely, for any sequence $\left(a_{k}\right)_{k \in \mathbb{Z}}$ vanishing at infinity, consider the

$$
h(x)=\sum_{k \in \mathbb{Z}} a_{k} \Delta_{k}^{*}(x), \quad x \in \mathbb{R},
$$

and its partial sums

$$
h_{n}(x)=\sum_{|k| \leq n} a_{k} \Delta_{k}^{*}(x)
$$

For fixed $x$ there are at most two non-null terms in the series (recall that the supports of $\Delta_{k}^{*}$ and $\Delta_{k+1}^{*}$ overlap), so pointwise convergence is obvious and the function $h$ is well defined. Clearly,

$$
\left\|h-h_{n}\right\|_{\infty} \leq 2 \max _{|k|>n}\left|a_{k}\right| .
$$

Moreover, for any $x, y \in \mathbb{R}$,

$$
\begin{aligned}
\left|\left(h-h_{n}\right)(x)-\left(h-h_{n}\right)(y)\right| & \leq \sum_{|k|>n}\left|a_{k}\right| \cdot\left|\Delta_{k}^{*}(x)-\Delta_{k}^{*}(y)\right| \\
& \leq 4|x-y| \max _{|k|>n}\left|a_{k}\right|,
\end{aligned}
$$

since for fixed $x$ and $y$ there are at most four non-null terms on the right hand side of (17). It follows that

$$
w_{\alpha}\left(h-h_{n}, \delta\right) \leq 4 \delta^{1-\alpha} \max _{|k|>n}\left|a_{k}\right| .
$$

This estimate together with (16) gives the convergence of $h_{n}$ to $h$ in the norm topology of $C_{0}^{\alpha}$. Hence $h$ belongs to $V_{0}$ and (i) is established.

The proof of (ii) is similar, the only differences being the dyadic change of scale and the non-overlapping of the supports of $\Delta_{j, k}$ and $\Delta_{j, k+1}$.

REMARK. As a by-product of (i) and (ii), the following geometric description of the spaces $V_{0}$ and $W_{j}$ is worth noticing.

- $V_{0}$ is the space of polygonal lines vanishing at infinity, with nodes at the integers $k \in \mathbb{Z}$.

- $W_{j}$ is the space of polygonal lines vanishing at infinity, with nodes at the points $l 2^{-j-1}(l \in \mathbb{Z})$ and vanishing at the points $k 2^{-j}(k \in \mathbb{Z})$.

- For $j \geq 1$, the direct sum $V_{j}:=V_{0} \oplus\left(\bigoplus_{i=0}^{j-1} W_{i}\right)$ is the space of polygonal lines vanishing at infinity, with nodes at the points $k 2^{-j}(k \in \mathbb{Z})$.

Proof of Theorem 1. Let $f \in C_{0}^{\alpha, 0}$. Recall that $E_{j} f$ is the linear interpolation of $f$ with nodes at the $r_{j, k}=k 2^{-j}$. By the geometrical description above, we clearly have $E_{0} f \in V_{0}$ and $\left(E_{j+1}-E_{j}\right) f \in W_{j}$. Now

$$
E_{j+1} f=E_{0} f+\sum_{i=0}^{j}\left(E_{i+1}-E_{i}\right) f
$$


and, by (5) and (6), this expression converges to $f$. This gives the decomposition

$$
f=E_{0} f+\sum_{j=0}^{\infty}\left(E_{j+1}-E_{j}\right) f \quad\left(\text { strong convergence in } C_{0}^{\alpha}\right) .
$$

The decomposition (13) of $E_{0} f$ is obvious by Lemma 2(i). Applying (ii) of the same lemma to $g=D_{j} f=\left(E_{j+1}-E_{j}\right) f$, we find that the corresponding coefficients $a_{j, k}(g)$ are

$$
\begin{aligned}
a_{j, k}(g) & =E_{j+1} f\left(\left(k+\frac{1}{2}\right) 2^{-j}\right)-E_{j} f\left(\left(k+\frac{1}{2}\right) 2^{-j}\right) \\
& =f\left(\left(k+\frac{1}{2}\right) 2^{-j}\right)-\frac{1}{2}\left\{f\left(k 2^{-j}\right)+f\left((k+1) 2^{-j}\right)\right\},
\end{aligned}
$$

since $E_{j} f$ is affine on the segment $\left[k 2^{-j},(k+1) 2^{-j}\right]$. So (14) is verified. Finally, from (6) we get the estimate

$$
\left\|E_{j} f\right\|_{\alpha} \leq 6\|f\|_{\alpha}, \quad f \in C_{0}^{\alpha, 0},
$$

from which the continuity of the projections $E_{j}$ and $D_{j}=E_{j+1}-E_{j}$ follows.

We now turn to the characterization of the spaces $C_{0}^{\alpha}$ and $C_{0}^{\alpha, 0}$ by their isomorphism with some sequence spaces.

Theorem 3. Define $\mathcal{S}^{\alpha}$ as the space of doubly indexed sequences $u=$ $\left(u_{j, k}: j \geq-1, k \in \mathbb{Z}\right)$ such that for all $j \geq-1, \lim _{|k| \rightarrow \infty} u_{j, k}=0$ and

$$
\|u\|:=\sup _{j \geq-1} 2^{(j+1) \alpha} \sup _{k \in \mathbb{Z}}\left|u_{j, k}\right|<\infty .
$$

Then the operator $T: C_{0}^{\alpha} \rightarrow \mathcal{S}^{\alpha}$ defined by $T f=u$, where

$$
\begin{aligned}
u_{-1, k} & =f(k), \quad k \in \mathbb{Z}, \\
u_{j, k} & =f\left(\left(k+\frac{1}{2}\right) 2^{-j}\right)-\frac{1}{2}\left\{f\left(k 2^{-j}\right)+f\left((k+1) 2^{-j}\right)\right\}, \quad j \geq 0, k \in \mathbb{Z},
\end{aligned}
$$

is an isomorphism of Banach spaces.

Pr o of. First, $T$ maps continuously $C_{0}^{\alpha}$ into the Banach space $\left(\mathcal{S}^{\alpha},\|\|\right)$. This clearly follows from the estimates $\left|u_{-1, k}\right| \leq\|f\|_{\infty}$ and, for any $j \geq 0$, $\left|u_{j, k}\right| \leq 2^{-(j+1) \alpha} w_{\alpha}\left(f, 2^{-j-1}\right)$, which give $\|T f\| \leq\|f\|_{\alpha}$. by

Next, the natural candidate to invert $T$ is the operator $R$ given formally

$$
(R u)(x)=\sum_{k \in \mathbb{Z}} u_{-1, k} \Delta_{k}^{*}(x)+\sum_{j=0}^{\infty} \sum_{k \in \mathbb{Z}} u_{j, k} \Delta_{j, k}(x), \quad u \in \mathcal{S}^{\alpha}, x \in \mathbb{R} .
$$

Write $|u|$ for the sequence obtained by replacing each term of $u$ by its absolute value. Since for fixed $x$ there are at most two non-vanishing $\Delta_{k}^{*}(x)$ and 
one $\Delta_{j, k}(x)$ (for each $j$ ), we get

$$
\begin{aligned}
R|u|(x) & \leq 2 \sup _{k \in \mathbb{Z}}\left|u_{-1, k}\right|+\sum_{j=0}^{\infty} 2^{-(j+1) \alpha} \sup _{k \in \mathbb{Z}} 2^{(j+1) \alpha}\left|u_{j, k}\right| \\
& \leq 2 \sup _{k \in \mathbb{Z}}\left|u_{-1, k}\right|+\frac{1}{2^{\alpha}-1} \sup _{j \geq 0} \sup _{k \in \mathbb{Z}} 2^{(j+1) \alpha}\left|u_{j, k}\right| .
\end{aligned}
$$

From this it follows at once that the function $R u$ is well defined, continuous on $\mathbb{R}$ and vanishes at infinity. Moreover,

$$
\|R u\|_{\infty} \leq \frac{2}{2^{\alpha}-1}\|u\|
$$

To check the Hölder regularity of $R u$, fix $x, y \in \mathbb{R}$ such that $0<|x-y|<1$ and write

$$
|R u(x)-R u(y)| \leq \sum_{k \in \mathbb{Z}}\left|u_{-1, k}\right| \cdot\left|\Delta_{k}^{*}(x)-\Delta_{k}^{*}(y)\right|+\sum_{j=0}^{\infty} A_{j}
$$

with

$$
A_{j}=\sum_{k \in \mathbb{Z}}\left|u_{j, k}\right| \cdot\left|\Delta_{j, k}(x)-\Delta_{j, k}(y)\right| .
$$

The first series on the right hand side of (26), having at most four nonvanishing terms, can be bounded by

$$
\sum_{k \in \mathbb{Z}}\left|u_{-1, k}\right| \cdot\left|\Delta_{k}^{*}(x)-\Delta_{k}^{*}(y)\right| \leq 4 \sup _{k \in \mathbb{Z}}\left|u_{-1, k}\right| \cdot|x-y| .
$$

Since $0 \leq \Delta_{j, k} \leq 1$ and the maximal slope of $\Delta_{j, k}$ is $2^{j+1}$, the estimates

$$
\left|\Delta_{j, k}(x)-\Delta_{j, k}(y)\right| \leq \min \left(1,2^{j+1}|x-y|\right), \quad k \in \mathbb{Z},
$$

provide the bound

$$
A_{j} \leq 2 \sup _{k \in \mathbb{Z}}\left|u_{j, k}\right| \min \left(1,2^{j+1}|x-y|\right) .
$$

Let $j_{0}$ be the integer defined by $2^{-j_{0}-1} \leq|x-y|<2^{-j_{0}}$. Splitting the series $\sum_{j \geq 0} A_{j}$ in two sums indexed by $j \leq j_{0}$ and $j>j_{0}$ and using (29) we obtain

$$
\begin{gathered}
\sum_{j=0}^{\infty} A_{j} \leq 2 \sup _{i \leq j_{0}, k \in \mathbb{Z}} 2^{(i+1) \alpha}\left|u_{i, k}\right| \sum_{j=0}^{j_{0}} 2^{(j+1)(1-\alpha)}|x-y| \\
+2 \sup _{i>j_{0}, k \in \mathbb{Z}} 2^{(i+1) \alpha}\left|u_{i, k}\right| \sum_{j=j_{0}+1}^{\infty} 2^{-(j+1) \alpha} .
\end{gathered}
$$


The elementary bounds

$$
\sum_{j=0}^{j_{0}} 2^{(j+1)(1-\alpha)} \leq \frac{2^{2-\alpha}}{2-2^{\alpha}}|x-y|^{\alpha-1}
$$

and

$$
\sum_{j=j_{0}+1}^{\infty} 2^{-(j+1) \alpha} \leq \frac{1}{2^{\alpha}-1}|x-y|^{\alpha}
$$

give

$$
\sum_{j=0}^{\infty} A_{j} \leq\left(\frac{8}{2-2^{\alpha}}+\frac{2}{2^{\alpha}-1}\right)\|u\| \cdot|x-y|^{\alpha} .
$$

Finally from (25), (28) and (32) we get

$$
\|R u\|_{\alpha} \leq\left(4+\frac{8}{2-2^{\alpha}}+\frac{4}{2^{\alpha}-1}\right)\|u\| .
$$

Hence the function $R u$ belongs to $C_{0}^{\alpha}$, the operator $R$ is continuous and since $R \circ T$ is the identity of $C_{0}^{\alpha}$, this completes the proof.

Remark. It is now easy to see that $C_{0}^{\alpha}$ is not separable. For instance, it contains the closed subspace

$$
L:=\left\{f=\sum_{j=0}^{\infty} \frac{v_{j}}{2^{(j+1) \alpha}} \Delta_{j, 0}:\left(v_{j}\right)_{j \geq 0} \in \ell^{\infty}(\mathbb{N})\right\},
$$

which is isomorphic to $\ell^{\infty}(\mathbb{N})$.

Theorem 4. $C_{0}^{\alpha, 0}$ is isomorphic via $T$ to the subspace $\mathcal{S}^{\alpha, 0}$ of $\mathcal{S}^{\alpha}$ defined by

$$
\mathcal{S}^{\alpha, 0}:=\left\{u \in \mathcal{S}^{\alpha}: \lim _{j \rightarrow \infty} 2^{j \alpha} \sup _{k \in \mathbb{Z}}\left|u_{j, k}\right|=0\right\} .
$$

Proof. By Theorem 3, it suffices to prove the inclusions $T\left(C_{0}^{\alpha, 0}\right) \subset \mathcal{S}^{\alpha, 0}$ and $R\left(\mathcal{S}^{\alpha, 0}\right) \subset C_{0}^{\alpha, 0}$. If $u=T f$ with $f \in C_{0}^{\alpha, 0}$, then by the definition of $u_{j, k}$ we have

$$
\left|u_{j, k}\right| \leq 2^{-(j+1) \alpha} w_{\alpha}\left(f, 2^{-j-1}\right), \quad k \in \mathbb{Z}
$$

from which the first inclusion follows.

Conversely, for any $u \in \mathcal{S}^{\alpha, 0}$, consider the function $f=R u$. By (34), the sequence $\left(\varepsilon_{j}\right)_{j \geq 0}$ defined by

$$
\varepsilon_{j}:=2^{(j+1) \alpha} \sup _{k \in \mathbb{Z}}\left|u_{j, k}\right|
$$

vanishes at infinity. To verify that $w_{\alpha}(f, \delta)$ decreases to zero with $\delta$, there is no loss of generality in assuming $\delta$ of the form $2^{-l}$. For $x, y \in \mathbb{R}$ such that $0<|x-y|<\delta$, let $j_{0}$ be the integer such that $2^{-j_{0}-1} \leq|x-y|<2^{-j_{0}}$. 
Using (28) and (29) with the same splitting as in the proof of Theorem 3 we obtain

$$
\begin{aligned}
\frac{|f(x)-f(y)|}{|x-y|^{\alpha}} \leq & 4 \sup _{k \in \mathbb{Z}}\left|u_{-1, k}\right| \cdot|x-y|^{1-\alpha} \\
& +2 \sum_{j=0}^{j_{0}} \varepsilon_{j} 2^{(j+1)(1-\alpha)}|x-y|^{1-\alpha} \\
& +2 \sum_{j=j_{0}+1}^{\infty} \varepsilon_{j} 2^{-(j+1) \alpha}|x-y|^{-\alpha} \\
\leq & 4\|u\| \delta^{1-\alpha}+4 \sum_{j=0}^{j_{0}} \varepsilon_{j} 2^{\left(j-j_{0}\right)(1-\alpha)}+2 \sum_{j=j_{0}+1}^{\infty} \varepsilon_{j} 2^{\left(j_{0}-j\right) \alpha} \\
\leq & 4\|u\| \delta^{1-\alpha}+4 v_{j_{0}}+\frac{4}{2^{\alpha}-1} \sup _{j \geq j_{0}} \varepsilon_{j},
\end{aligned}
$$

with

$$
v_{j_{0}}:=\sum_{i=0}^{j_{0}} \varepsilon_{j_{0}-i} 2^{i(\alpha-1)} .
$$

By the Lebesgue dominated convergence theorem for series, $\lim _{j_{0} \rightarrow \infty} v_{j_{0}}$ $=0$. Taking the supremum over $x, y$ in the above estimates we obtain (recalling that $\delta=2^{-l}$ )

$$
w_{\alpha}(f, \delta) \leq 4\|u\| \delta^{1-\alpha}+4 \sup _{j \geq l} v_{j}+\frac{4}{2^{\alpha}-1} \sup _{j \geq l} \varepsilon_{j} .
$$

Hence $w_{\alpha}(f, \delta)$ decreases to zero with $\delta$. The inclusion $R\left(\mathcal{S}^{\alpha, 0}\right) \subset C_{0}^{\alpha, 0}$ is proven and hence also the theorem.

We end this section by exploiting the isomorphism with sequence spaces to find some representations of the topological dual of $C_{0}^{\alpha, 0}$.

Lemma 5. Set $\mathbb{I}=(\{-1\} \cup \mathbb{N}) \times \mathbb{Z}$. Then $\psi$ is a continuous linear functional on $\mathcal{S}^{\alpha, 0}$ if and only if, for some $z \in \ell^{1}(\mathbb{I})$,

$$
\psi(u)=\sum_{j \geq 1} \sum_{k \in \mathbb{Z}} 2^{(j+1) \alpha} u_{j, k} z_{j, k}, \quad u \in \mathcal{S}^{\alpha, 0} .
$$

This representation is unique.

Proof. One can verify that the family $\left\{e_{j, k}^{(\alpha)}:(j, k) \in \mathbb{I}\right\}$ defined by

$$
e_{j, k}^{(\alpha)}(i, l)= \begin{cases}2^{-(j+1) \alpha} & \text { if }(i, l)=(j, k), \\ 0 & \text { otherwise }\end{cases}
$$


is an unconditional basis of $\mathcal{S}^{\alpha, 0}$. From this point on, the proof follows the same lines as the classical proof of the duality between $c_{0}(\mathbb{N})$ and $\ell^{1}(\mathbb{N})$ at the cost of some slight complications due to the double indexation.

Lemma 5 and the isomorphism between $C_{0}^{\alpha, 0}$ and $\mathcal{S}^{\alpha, 0}$ give a sequential characterization of the dual of $\mathcal{C}_{0}^{\alpha, 0}$ :

Theorem 6. The functional $\varphi$ belongs to the topological dual of $C_{0}^{\alpha, 0}$ if and only if, for some $z \in \ell^{1}(\mathbb{I})$,

$$
\varphi(f)=\sum_{j \geq-1} \sum_{k \in \mathbb{Z}} 2^{(j+1) \alpha} z_{j, k} u_{j, k}(f), \quad f \in C_{0}^{\alpha, 0},
$$

where the linear functionals $u_{j, k}(f)$ are given by the isomorphism $T$ of Theorem 3 . This representation is unique.

Finally, we can give a more intrinsic representation of the dual.

TheOREM 7. $\varphi$ is a continuous linear functional on $C_{0}^{\alpha, 0}$ if and only if there exist a signed measure $\mu$ on $\mathbb{R}$ and a signed measure $\nu$ on $\mathbb{R} \times[0,1]$ such that

$$
\begin{aligned}
\varphi(f)= & \int_{\mathbb{R}} f(x) \mu(d x) \\
& +\int_{\mathbb{R} \times[0,1]} \frac{2 f(x)-f(x+y)-f(x-y)}{y^{\alpha}} \nu(d x, d y),
\end{aligned}
$$

where the second integrand is defined to be 0 when $y=0$, which amounts to an extension by continuity since $f \in C_{0}^{\alpha, 0}$.

Proof. Clearly, if $\varphi$ is defined by (39), then

$$
\begin{aligned}
|\varphi(f)| & \leq\|f\|_{\infty}|\mu|(\mathbb{R})+2 w_{\alpha}(f, 1)|\nu|(\mathbb{R} \times[0,1]) \\
& \leq\{|\mu|(\mathbb{R})+2|\nu|(\mathbb{R} \times[0,1])\}\|f\|_{\alpha} .
\end{aligned}
$$

Conversely, if $\varphi$ is a linear continuous functional on $C_{0}^{\alpha, 0}$, then using the representation (38) and choosing

$$
\mu=\sum_{k \in \mathbb{Z}} z_{-1, k} \delta_{k} \quad \text { and } \quad \nu=\sum_{j \geq 0} \sum_{k \in \mathbb{Z}} \frac{1}{2} z_{j, k} \delta_{k 2^{-j}} \otimes \delta_{2^{-j-1}}
$$

we directly obtain (39).

3. Tightness in $C_{0}^{\alpha, 0}$. We present here some conditions for tightness of sequences of stochastic processes with paths in $C_{0}^{\alpha, 0}$. Our main tool is the following theorem which is a generalization of Prokhorov's theorem [12] about tightness in Hilbert spaces. 
Theorem 8 (Suquet [16]). Let $\mathcal{X}$ be a separable Banach space having a Schauder decomposition

$$
\left.\mathcal{X}=\bigoplus_{i=0}^{\infty} \mathcal{X}_{i} \quad \text { (topological direct sum }\right)
$$

Write

$$
V_{j}=\bigoplus_{i=0}^{j} \mathcal{X}_{i}, \quad j=0,1, \ldots,
$$

and denote by $E_{j}$ the continuous projection from $\mathcal{X}$ onto $V_{j}$. Let $\mathcal{F}$ be a family of probability measures on $\mathcal{X}$ and $E_{j} \mathcal{F}=\left\{\mu \circ E_{j}^{-1}: \mu \in \mathcal{F}\right\}$. Then $\mathcal{F}$ is tight if and only if:

(i) $E_{j} \mathcal{F}$ is tight for $j=0,1, \ldots$,

(ii) for each positive $\varepsilon$,

$$
\lim _{j \rightarrow \infty} \sup _{\mu \in \mathcal{F}} \mu\left(f \in \mathcal{X}:\left\|f-E_{j} f\right\|>\varepsilon\right)=0 .
$$

REMARK. It is easily seen that $K$ is compact in $V_{j}$ if and only if $\pi_{i} K$ is compact in $\mathcal{X}_{i}(0 \leq i \leq j)$ where $\pi_{i}$ is the canonical projection on $\mathcal{X}_{i}$. Thus condition (i) can be replaced by the following one which is more convenient in our setting:

(i') $\pi_{i} \mathcal{F}$ is tight for $i=0,1,2, \ldots$

Our first result is a necessary and sufficient condition for tightness, based on the isomorphism between $C_{0}^{\alpha, 0}$ and the sequence space $\mathcal{S}^{\alpha, 0}$.

THEOREM 9. Let $\left(\xi_{n}: n \geq 1\right)$ be a sequence of random elements in $C_{0}^{\alpha, 0}$. Define the random variables $u_{j, k}\left(\xi_{n}\right)(j \geq-1, k \in \mathbb{Z})$ by

$$
\begin{aligned}
u_{-1, k}\left(\xi_{n}\right) & =\xi_{n}(k), \\
u_{j, k}\left(\xi_{n}\right) & =\xi_{n}\left(\left(k+\frac{1}{2}\right) 2^{-j}\right)-\frac{1}{2}\left\{\xi_{n}\left(k 2^{-j}\right)+\xi_{n}\left((k+1) 2^{-j}\right)\right\}, \quad j \geq 0 .
\end{aligned}
$$

Then $\left(\xi_{n}: n \geq 1\right)$ is tight in $C_{0}^{\alpha, 0}$ if and only if the following three conditions are satisfied:

$$
\begin{gathered}
\lim _{A \rightarrow \infty} \sup _{n \geq 1} P\left(\left|u_{i, k}\left(\xi_{n}\right)\right| \geq A\right)=0, \quad i \geq-1, k \in \mathbb{Z}, \\
\lim _{q \rightarrow \infty} \sup _{n \geq 1} P\left(\sup _{|k|>q}\left|u_{i, k}\left(\xi_{n}\right)\right| \geq \varepsilon\right)=0, \quad i \geq-1, \quad \varepsilon>0, \\
\lim _{j \rightarrow \infty} \sup _{n \geq 1} P\left(\sup _{i \geq j} 2^{(i+1) \alpha} \sup _{k \in \mathbb{Z}}\left|u_{i, k}\left(\xi_{n}\right)\right| \geq \varepsilon\right)=0, \quad \varepsilon>0 .
\end{gathered}
$$

Proof. By the Schauder decomposition (12) from Theorem 1 and the isomorphism $T,(42)$ appears as a simple rephrasing of condition (ii) of Theorem 8. To see that condition (i') for $\mathcal{X}=C_{0}^{\alpha, 0}$ is equivalent to (40) and (41) it suffices to apply again Theorem 8 but to the space $T\left(V_{0}\right)$ or 
$T\left(W_{i-1}\right)(i \geq 1)$, the Schauder decomposition being in this case given by the canonical Schauder basis of the corresponding space.

From Theorem 9, we can deduce the following characterization of tightness which has a more intrinsic form.

Theorem 10. A sequence $\left(\xi_{n}: n \geq 1\right)$ of random elements in $C_{0}^{\alpha, 0}$ is tight if and only if the following two conditions are satisfied:

(a) For any positive $\varepsilon, \lim _{A \rightarrow \infty} \sup _{n \geq 1} P\left(\sup _{|t|>A}\left|\xi_{n}(t)\right| \geq \varepsilon\right)=0$.

(b) For any positive $\varepsilon, \lim _{\delta \rightarrow 0} \sup _{n \geq 1} P\left(w_{\alpha}\left(\xi_{n}, \delta\right) \geq \varepsilon\right)=0$.

Proof. Obviously, (a) implies (41). By the estimates (5), (6) and the isomorphism $T$, (42) follows from (b). Let us see how (40) follows from (a) and (b). The case $i \geq 0$ is obvious since

$$
\left|u_{i, k}\left(\xi_{n}\right)\right| \leq 2 w_{\alpha}\left(\xi_{n}, 2^{-i-1}\right) 2^{-(i+1) \alpha} .
$$

To handle the case $i=-1$, fix $\eta>0$. With $\varepsilon=1$ in (a), we get, for some $t_{0}$ in $\mathbb{R}$,

$$
\sup _{n \geq 1} P\left(\left|\xi_{n}\left(t_{0}\right)\right| \geq 1\right)<\eta .
$$

By (b) there is some $\delta>0$ such that

$$
P\left(w_{\alpha}\left(\xi_{n}, \delta\right)<1\right) \geq 1-\eta \quad(n \geq 1) .
$$

For each $k$ in $\mathbb{Z}$ let $N$ be the integer defined by $(N-1) \delta \leq\left|k-t_{0}\right|<N \delta$. By chaining we obtain

$$
P\left(\left|\xi_{n}(k)-\xi_{n}\left(t_{0}\right)\right| \leq N \delta^{\alpha}\right) \geq 1-2 \eta \quad(n \geq 1) .
$$

Hence for $A=1+N \delta^{\alpha}$ we have

$$
P\left(\left|\xi_{n}(k)\right| \geq A\right) \leq 3 \eta \quad(n \geq 1),
$$

which ends the verification of the case $i=-1$.

So conditions (a) and (b) are sufficient for the tightness of $\left(\xi_{n}: n \geq 1\right)$ in $C_{0}^{\alpha, 0}$. To prove their necessity, we use the following

LEMma 11 (Suquet [16]). Let $\mathcal{F}$ be a compact family (in the topology of weak convergence) of probability measures on the separable metric space $S$. Let $\left(F_{l}: l \in \mathbb{N}\right)$ be a sequence of closed subsets of $S$ decreasing to $\emptyset$. Define the functions $\varphi_{l}(l \in \mathbb{N})$ by $\varphi_{l}: \mathcal{F} \rightarrow \mathbb{R}^{+}, P \mapsto \varphi_{l}(P)=P\left(F_{l}\right)$. Then the sequence $\left(\varphi_{l}\right)$ converges to zero uniformly on $\mathcal{F}$.

Now consider two sequences $A_{l} \uparrow \infty, \delta_{l} \downarrow 0$ and define, for fixed $\varepsilon>0$,

$$
\begin{aligned}
& F_{l}^{(a)}:=\left\{f \in C_{0}^{\alpha, 0}: \sup _{|s| \geq A_{l}}|f(s)| \geq \varepsilon\right\} \\
& F_{l}^{(b)}:=\left\{f \in C_{0}^{\alpha, 0}: w_{\alpha}\left(f, \delta_{l}\right) \geq \varepsilon\right\} .
\end{aligned}
$$


By the obvious continuity on $C_{0}^{\alpha, 0}$ of the functionals involved in their definitions, these sets are closed, so applying Lemma 11 we obtain the necessity of conditions (a) and (b) for the tightness of $\left(\xi_{n}: n \geq 1\right)$.

We now give a sufficient condition of practical use for the tightness in $C_{0}^{\alpha}$.

THEOREM 12. Let $\left(\xi_{n}: n \geq 1\right)$ be a sequence of random elements in $C_{0}^{\alpha, 0}$ and suppose that the following conditions are satisfied:

(i) For each $k \in \mathbb{Z}, \lim _{A \rightarrow \infty} \sup _{n \geq 1} P\left(\left|\xi_{n}(k)\right| \geq A\right)=0$.

(ii) For each $\varepsilon>0, \lim _{q \rightarrow \infty} \sup _{n \geq 1} P\left(\sup _{|k|>q}\left|\xi_{n}(k)\right| \geq \varepsilon\right)=0$.

(iii) There is a sequence of non-decreasing bounded functions $G_{n}$ and some constants $\gamma \geq 1, \delta>0$ such that

$$
P\left(\left|\xi_{n}(t)-\xi_{n}(s)\right| \geq \lambda\right) \leq \frac{|t-s|^{\delta}\left(G_{n}(t)-G_{n}(s)\right)}{\lambda^{\gamma}}
$$

for any $\lambda>0$ and $-\infty<s<t \leq s+1<\infty$.

(iv) $M:=\sup _{n \geq 1}\left(G_{n}(\infty)-G_{n}(-\infty)\right)<\infty$.

(v) The series $\sum_{l \in \mathbb{Z}}\left(G_{n}(l+1)-G_{n}(l)\right)$ converge uniformly in $n \geq 1$.

Then $\left(\xi_{n}: n \geq 1\right)$ is tight in $C_{0}^{\alpha, 0}$ for any $0<\alpha<\delta / \gamma$.

Proof. We check conditions (40)-(42) of Theorem 9. Conditions (i) and (ii) are a simple rephrasing of (40) and (41) in the special case $i=-1$.

Since for non-negative $i$,

$u_{i, k}\left(\xi_{n}\right)=\frac{1}{2}\left[\xi_{n}\left(\frac{k+1 / 2}{2^{i}}\right)-\xi_{n}\left(\frac{k}{2^{i}}\right)\right]-\frac{1}{2}\left[\xi_{n}\left(\frac{k+1}{2^{i}}\right)-\xi_{n}\left(\frac{k+1 / 2}{2^{i}}\right)\right]$,

condition (iii) provides the estimates

$$
P\left(\left|u_{i, k}\left(\xi_{n}\right)\right| \geq A\right) \leq \frac{1}{2 A^{\gamma} 2^{(i+1) \delta}}\left[G_{n}\left(\frac{k+1}{2^{i}}\right)-G_{n}\left(\frac{k}{2^{i}}\right)\right] .
$$

The $G_{n}$ 's being non-decreasing, condition (iv) gives the crude but sufficient inequality

$$
P\left(\left|u_{i, k}\left(\xi_{n}\right)\right| \geq A\right) \leq \frac{M}{2^{(i+1) \delta}} A^{-\gamma},
$$

which provides the verification of (40).

Next, to verify (41), we can assume without loss of generality that the integer $q$ has the form $q=m 2^{i}$ where $m$ is a positive integer going to infinity. Using the estimates (43) we obtain

$$
\begin{aligned}
& P\left(\sup _{|k|>q}\left|u_{i, k}\left(\xi_{n}\right)\right| \geq \varepsilon\right) \\
& \quad \leq \frac{1}{\varepsilon^{\gamma} 2^{(i+1) \delta}}\left\{\left[G_{n}(\infty)-G_{n}(m)\right]+\left[G_{n}(-m)-G_{n}(-\infty)\right]\right\} .
\end{aligned}
$$


By condition (v), this bound goes to zero uniformly in $n$ as $m \rightarrow \infty$. Hence (41) is verified.

Finally, to verify (42), the same method leads via (iv) to the upper bound

$$
\sup _{n \geq 1} P\left(\sup _{i \geq j} 2^{(i+1) \alpha} \sup _{k \in \mathbb{Z}}\left|u_{i, k}\left(\xi_{n}\right)\right| \geq \varepsilon\right) \leq \sum_{i \geq j} \frac{2^{(i+1)(\gamma \alpha-\delta)}}{\varepsilon^{\gamma}} M,
$$

which goes to zero as $j \rightarrow \infty$, subject to $\gamma \alpha-\delta<0$.

Comments. Of course, conditions (i) and (ii) can be replaced respectively by the following moment versions with some exponent $\tau>0$ :

(i') For each $k \in \mathbb{Z}, \sup _{n>1} \mathbb{E}\left|\xi_{n}(k)\right|^{\tau}<\infty$.

(ii') The series $\sum_{k \in \mathbb{Z}} \mathbb{E}\left|\xi_{n}(k)\right|^{\tau}$ converge uniformly in $n \geq 1$.

When dealing with random elements in $C_{0}^{\alpha, 0}$ generated by discontinuous processes via some smoothing procedure, condition (iii) seems sometimes too strong a requirement. Fortunately, it can be relaxed in the following way.

Corollary 13. Suppose the sequence $\left(\xi_{n}: n \geq 1\right)$ of random elements in $C_{0}^{\alpha, 0}$ satisfies

$$
\lim _{n \rightarrow \infty} w_{\alpha}\left(\xi_{n}, 2^{-j(n)}\right)=0 \quad \text { in probability, }
$$

where $j(n)$ is some sequence of integers increasing to infinity. Then Theorem 12 remains valid with condition (iii) satisfied only for $1 \geq t-s \geq 2^{-j(n)}$.

Proof. Consider the auxiliary processes $\widetilde{\xi}_{n}=E_{j(n)} \xi_{n}$. Since $u_{i, k}\left(\widetilde{\xi}_{n}\right)=$ 0 for $i>j(n)$, the proof of Theorem 12 gives the tightness of $\left(\widetilde{\xi}_{n}: n \geq\right.$ 1). Now, by (5), (6) and (46), $\widetilde{\xi}_{n}-\xi_{n} \rightarrow 0$ in probability. Hence for any subsequence $\left(\widetilde{\xi}_{n_{l}}: l \geq 1\right)$ converging in distribution in $C_{0}^{\alpha, 0},\left(\xi_{n_{l}}\right.$ : $l \geq 1)$ converges to the same limit. The result follows by the sequential characterization of tightness.

4. Application. We now present an application to the weak Hölder convergence of the so-called perturbed empirical process. Let $\left(X_{n}\right)_{n \geq 1}$ be a sequence of independent identically distributed real-valued random variables with marginal distribution function $F$. Write $F_{n}$ for the empirical distribution function

$$
F_{n}(t)=\frac{1}{n} \sum_{i=1}^{n} \mathbf{1}_{\left[X_{i}, \infty\right)}(t), \quad t \in \mathbb{R}
$$

and

$$
\xi_{n}(t)=\sqrt{n}\left(F_{n}(t)-F(t)\right), \quad t \in \mathbb{R},
$$


for the empirical process of the sample $\left(X_{1}, \ldots, X_{n}\right)$. We introduce a sequence of convolution kernels $K_{n}(t)=c_{n}^{-1} K\left(t / c_{n}\right)$ where $K$ is a probability density on the line and the parameter $c_{n}$ decreases to zero at a rate to be made precise later. The sequence $\left(K_{n}: n \geq 1\right)$ is an approximate identity, that is,

$$
\begin{array}{rlrl}
\int_{\mathbb{R}} K_{n}(t) d t & =1, & & n \geq 1, \\
\lim _{n \rightarrow \infty} \int_{|t| \geq \varepsilon} K_{n}(t) d t & =0, \quad & \varepsilon>0 .
\end{array}
$$

The corresponding perturbed empirical process $\zeta_{n}$ is then defined by

$$
\zeta_{n}=\sqrt{n}\left(F_{n}-F\right) * K_{n} .
$$

We will impose some extra conditions on $K_{n}$ to ensure that any path of $\zeta_{n}$ belongs to $C_{0}^{1 / 2}$ and so to all the $C_{0}^{\alpha, 0}$ for $\alpha<1 / 2$ (since we do not expect more regularity for the limiting process of $\zeta_{n}$ ). These conditions are provided by the following

LEMMA 14. Let $f$ be a bounded (possibly discontinuous) measurable function vanishing at infinity and $K$ a convolution kernel satisfying, for some constants $0<\varrho \leq 1$ and $a(K)$,

$$
\begin{gathered}
K \in L^{1}(\mathbb{R}) \cap L^{1-\varrho}(\mathbb{R}), \\
|K(x)-K(y)| \leq a(K)|x-y|, \quad x, y \in \mathbb{R} .
\end{gathered}
$$

Then $f * K$ belongs to $C_{0}^{\varrho}$.

Proof. By standard arguments, $f * K$ is bounded and vanishes at infinity. The obvious estimate

$$
|f * K(x)-f * K(y)| \leq 2\|f\|_{\infty} a(K)^{\varrho} \int_{\mathbb{R}} K(u)^{1-\varrho} d u|x-y|^{\varrho}
$$

gives $w_{\varrho}(f * K, 1)<\infty$. In the special case $\varrho=1, L^{0}(\mathbb{R})$ denotes here the space of bounded measurable functions vanishing outside some compact set.

REMARK. In fact, we could completely avoid imposing conditions like (52) and (53). This would require an adaptation of the theorems of Section 3 to make them into conditions for a given random function to have paths in $C_{0}^{\alpha}$ with probability one. For simplicity we do not go further this way and keep our initial point of view of stochastic processes considered as random elements in some function space $C_{0}^{\alpha}$ and therefore with each path in $C_{0}^{\alpha}$.

Theorem 15. Assume that the marginal distribution function $F$ of the i.i.d. sequence $\left(X_{i}: i \geq 1\right)$ satisfies, for some constants $C>0$ and 
$0<r \leq 1$,

$$
|F(x)-F(y)| \leq C|x-y|^{r}, \quad x, y \in \mathbb{R} .
$$

Define the kernels $K_{n}(t)=c_{n}^{-1} K\left(t / c_{n}\right)$ where the probability density $K$ satisfies (52) with some $1 / 2 \leq \varrho \leq 1$ and (53). Suppose moreover that for $0<\alpha<r / 2, c_{n}=c_{n}(\alpha)$ decreases to zero in such a way that

$$
n^{\alpha / \varrho-1}=o\left(c_{n}\right) \text {. }
$$

Then the sequence $\left(\zeta_{n}: n \geq 1\right)$ of perturbed empirical processes defined by (51) is tight in $C_{0}^{\alpha, 0}$ for any $\alpha<r / 2$.

Proof. We shall use Corollary 13 with the sequence $j(n)$ given by $2^{j(n)} \leq n<2^{j(n)+1}$. We need to verify (i) to (v) of Theorem 12 (only the relaxed version for (iii)).

First, (i) follows from (i') which holds for $\tau=2$. Indeed, by Jensen's inequality and Fubini's theorem we get

$$
\begin{aligned}
\mathbb{E}\left|\int_{\mathbb{R}} \xi_{n}(t-u) K_{n}(u) d u\right|^{2} & \leq \int_{\mathbb{R}} \mathbb{E}\left|\xi_{n}(t-u)\right|^{2} K_{n}(u) d u \\
& =H * K_{n}(t), \quad t \in \mathbb{R},
\end{aligned}
$$

where $H=F(1-F)$. Now, with $H_{n}=H * K_{n}$, we obviously have

$$
\mathbb{E}\left|\zeta_{n}(t)\right|^{2} \leq H_{n}(t) \leq 1, \quad t \in \mathbb{R}, n \geq 1 .
$$

To verify (ii), let us introduce $\chi_{n}$, the $[0,1]$-indexed empirical process of the $F\left(X_{i}\right)$ 's, and $\tilde{\chi}_{n}$, the polygonal empirical process obtained by replacing the empirical distribution function of $\left(F\left(X_{1}\right), \ldots, F\left(X_{n}\right)\right)$ by the cumulative empirical frequencies polygon (see for instance Billingsley [2], p. 104). We shall bound $\zeta_{n}(t)$ in terms of $\widetilde{\chi}_{n}$ in order to exploit the known tightness of the sequence $\left(\widetilde{\chi}_{n}\right)$ on $C[0,1]$. Noting that $\xi_{n}(t)=\chi_{n}(F(t))$ and $\left\|\widetilde{\chi}_{n}-\chi_{n}\right\|_{\infty} \leq$ $n^{-1 / 2}$ we have

$$
\begin{aligned}
\left|\zeta_{n}(t)-\widetilde{\chi}_{n}(F(t))\right| \leq & \frac{1}{\sqrt{n}}+\int_{\mathbb{R}}\left|\widetilde{\chi}_{n}(F(t-u))-\widetilde{\chi}_{n}(F(t))\right| K_{n}(u) d u \\
\leq & \frac{1}{\sqrt{n}}+\int_{-1}^{1}\left|\widetilde{\chi}_{n}(F(t-u))-\widetilde{\chi}_{n}(F(t))\right| K_{n}(u) d u \\
& +2\left\|\widetilde{\chi}_{n}\right\|_{\infty} \int_{|u| \geq 1} K_{n}(u) d u \\
\leq & \frac{1}{\sqrt{n}}+w_{0}\left(\widetilde{\chi}_{n}, F(t+1)-F(t-1)\right) \\
& +2\left\|\widetilde{\chi}_{n}\right\|_{\infty} \int_{|u| \geq 1} K_{n}(u) d u,
\end{aligned}
$$


where $w_{0}$ denotes the classical modulus of continuity on $C[0,1]$. Since $\tilde{\chi}_{n}$ vanishes at 0 and 1 , we have $\widetilde{\chi}_{n}(F(t)) \leq \min \left(w_{0}\left(\widetilde{\chi}_{n}, F(t)\right), w_{0}\left(\widetilde{\chi}_{n}, 1-F(t)\right)\right)$, so finally

$$
\max _{|t| \geq q}\left|\zeta_{n}(t)\right| \leq 2 w_{0}\left(\widetilde{\chi}_{n}, \delta_{q}\right)+\frac{1}{\sqrt{n}}+2\left\|\widetilde{\chi}_{n}\right\|_{\infty} \int_{|u| \geq 1} K_{n}(u) d u,
$$

where $\delta_{q}=\max (F(-q+1), 1-F(q-1))$. The tightness of $\left(\widetilde{\chi}_{n}\right)$ on $C[0,1]$ then gives the boundedness in probability of the sequence $\left(\left\|\widetilde{\chi}_{n}\right\|_{\infty}: n \geq 1\right)$ and the uniform convergence in probability to zero of $w_{0}\left(\widetilde{\chi}_{n}, \delta_{q}\right)$ as $q \rightarrow \infty$. This together with (50) and the almost sure vanishing at infinity of the paths of each $\zeta_{n}$ gives (ii).

Since $1 / n \leq 2^{-j(n)}<2 / n$, Corollary 13 allows us to verify (iii) only for $1 \geq t-s \geq 1 / n$, provided that we can prove the convergence in probability to zero of $w_{\alpha}\left(\zeta_{n}, 2 / n\right)$. To begin with, Rosenthal's inequality gives for any $\gamma>2$ a constant $C_{\gamma}$ such that

$$
\mathbb{E}\left|\zeta_{n}(t)-\zeta_{n}(s)\right|^{\gamma} \leq C_{\gamma}\left[n^{1-\gamma / 2} \mathbb{E}\left|Y_{1}\right|^{\gamma}+\left(\mathbb{E} Y_{1}^{2}\right)^{\gamma / 2}\right],
$$

with the i.i.d. bounded centered random variables $Y_{i}$ defined as

$$
Y_{i}=\int_{\mathbb{R}}\left(\mathbf{1}_{(s-u, t-u]}\left(X_{i}\right)-\mathbb{E} \mathbf{1}_{(s-u, t-u]}\left(X_{i}\right)\right) K_{n}(u) d u .
$$

By Jensen's inequality, Fubini's theorem and elementary estimates of the moments of centered Bernoulli random variables, we obtain, for $s<t$ and $p \geq 2$,

$$
\begin{aligned}
\mathbb{E}\left|Y_{1}\right|^{p} & \leq \int_{\mathbb{R}} \mathbb{E}\left|\mathbf{1}_{(s-u, t-u]}\left(X_{i}\right)-\mathbb{E} \mathbf{1}_{(s-u, t-u]}\left(X_{i}\right)\right|^{p} K_{n}(u) d u \\
& \leq \int_{\mathbb{R}}(F(t-u)-F(s-u)) K_{n}(u) d u \\
& \leq F * K_{n}(t)-F * K_{n}(s) .
\end{aligned}
$$

Writing $\widetilde{G}_{n}$ for the distribution function $F * K_{n}$ and going back to (60), we get

$$
\begin{aligned}
\mathbb{E} \mid \zeta_{n}(t)- & \left.\zeta_{n}(s)\right|^{\gamma} \\
& \leq C_{\gamma}\left[n^{1-\gamma / 2}+\left(\widetilde{G}_{n}(t)-\widetilde{G}_{n}(s)\right)^{\gamma / 2-1}\right]\left(\widetilde{G}_{n}(t)-\widetilde{G}_{n}(s)\right) .
\end{aligned}
$$

Now, since the $K_{n}$ 's are probability densities, the $\widetilde{G}_{n}$ 's inherit the $r$-Hölder regularity of $F$ (see (55)) with the same constant $C$ independent of $n$. Moreover, for $1 \geq t-s \geq 1 / n$, we have $n^{1-\gamma / 2} \leq(t-s)^{\gamma / 2-1} \leq(t-s)^{r(\gamma / 2-1)}$. Hence for $1 \geq t-s \geq 1 / n$,

$$
\mathbb{E}\left|\zeta_{n}(t)-\zeta_{n}(s)\right|^{\gamma} \leq C_{\gamma}^{\prime}|t-s|^{r(\gamma / 2-1)}\left(\widetilde{G}_{n}(t)-\widetilde{G}_{n}(s)\right),
$$


with $C_{\gamma}^{\prime}=C_{\gamma}\left(1+C^{\gamma / 2-1}\right)$. So the relaxed version of (iii) is satisfied for any $\gamma>2$ with $\delta=r(\gamma / 2-1)$ and

$$
G_{n}:=C_{\gamma}^{\prime} F * K_{n} .
$$

Since the $F * K_{n}$ are distribution functions, (iv) is obviously satisfied.

To check (v), we drop the constant $C_{\gamma}^{\prime}$ and observe that for $m \in \mathbb{N}$,

$$
\begin{aligned}
& \sum_{l \geq m}\left(F * K_{n}(l+1)-F * K_{n}(l)\right)=(1-F) * K_{n}(m), \\
& \sum_{l \leq-m}\left(F * K_{n}(l)-F * K_{n}(l-1)\right)=F * K_{n}(-m) .
\end{aligned}
$$

The sequence $\left(K_{n}\right)$ being an approximate identity, $(1-F) * K_{n}$ and $F * K_{n}$ converge uniformly on $\mathbb{R}$ to $1-F$ and $F$ respectively. Hence the convergence to 0 as $m \rightarrow \infty$ of the right hand sides of (65) and (66) is uniform in $n$. This completes the verification of condition (v).

To complete the proof it remains to show the convergence in probability to zero of $w_{\alpha}\left(\zeta_{n}, 2 / n\right)$. For any pair $s<t$ we have

$$
\begin{aligned}
\frac{\left|\zeta_{n}(t)-\zeta_{n}(s)\right|}{|t-s|^{\alpha} \leq} & \int_{\mathbb{R}}\left|\xi_{n}(u)\right| \frac{\left|K_{n}(t-u)-K_{n}(s-u)\right|^{\varrho}}{|t-s|^{\alpha}} \\
& \times\left(K_{n}(t-u)+K_{n}(s-u)\right)^{1-\varrho} d u \\
\leq & a(K)^{\varrho} \frac{|t-s|^{\varrho-\alpha}}{c_{n}^{2 \varrho}}\left(1+\left\|\widetilde{\chi}_{n}\right\|_{\infty}\right) \\
& \times \int_{\mathbb{R}}\left(K_{n}(t-u)^{1-\varrho}+K_{n}(s-u)^{1-\varrho}\right) d u \\
\leq & 2 a(K)^{\varrho} \int_{\mathbb{R}} K(v)^{1-\varrho} d v\left(1+\left\|\widetilde{\chi}_{n}\right\|_{\infty}\right) \frac{|t-s|^{\varrho-\alpha}}{c_{n}^{\varrho}} .
\end{aligned}
$$

Recalling that $\varrho-\alpha$ is positive and taking the supremum over $|t-s| \leq 2 / n$, we obtain, with an obvious constant $C_{\varrho, K}$,

$$
w_{\alpha}\left(\zeta_{n}, 2 / n\right) \leq C_{\varrho, K}\left(1+\left\|\widetilde{\chi}_{n}\right\|_{\infty}\right) \frac{n^{\alpha-\varrho}}{c_{n}^{\varrho}} .
$$

By the tightness of $\left(\left\|\widetilde{\chi}_{n}\right\|_{\infty}: n \geq 1\right)$ and (56), this last upper bound goes to zero in probability.

Finally, by Corollary 13 , for any $\alpha<r(\gamma / 2-1) / \gamma$, the sequence $\left(\zeta_{n}\right.$ : $n \geq 1)$ is tight in $C_{0}^{\alpha, 0}$. As there is no upper bound on the choice of $\gamma$ in the moment inequality (60) and $\gamma$ is not involved in the constraints on $c_{n}$, the tightness holds for any $\alpha<r / 2$ (of course, subject to the constraint (56) on the sequence $\left.\left(c_{n}\right)\right)$. 
THEOREM 16. Under the assumptions of Theorem 15 the sequence $\left(\zeta_{n}\right.$ : $n \geq 1)$ of perturbed empirical processes defined by (51) is weakly convergent in $C_{0}^{\alpha, 0}$ for any $0<\alpha<r / 2$ to a centered Gaussian process $\zeta$ with covariance function

$$
\Gamma(s, t)=F(s) \wedge F(t)-F(s) F(t), \quad s, t \in \mathbb{R} .
$$

Proof. By Theorem 15, $\left(\zeta_{n}: n \geq 1\right)$ is tight in $C_{0}^{\alpha, 0}$ for any $0<\alpha<r / 2$. To check the convergence of the finite-dimensional distributions of $\zeta_{n}$, write

$$
\zeta_{n}=\xi_{n}+\left(\xi_{n} * K_{n}-\xi_{n}\right)
$$

and recall that the finite-dimensional distributions of $\xi_{n}$ converge to those of $\zeta$ by the multinomial central limit theorem. So it suffices to prove that for each $t,\left(\xi_{n} * K_{n}-\xi_{n}\right)(t) \rightarrow 0$ in probability. By Jensen's inequality,

$$
\left|\xi_{n} * K_{n}(t)-\xi_{n}(t)\right|^{2} \leq \int_{\mathbb{R}}\left|\xi_{n}(t-u)-\xi_{n}(t)\right|^{2} K_{n}(u) d u .
$$

Hence we have the variance estimates

$$
\begin{aligned}
\mathbb{E}\left|\xi_{n} * K_{n}(t)-\xi_{n}(t)\right|^{2} & \leq \int_{\mathbb{R}} \mathbb{E}\left|\xi_{n}(t-u)-\xi_{n}(t)\right|^{2} K_{n}(u) d u \\
& \leq \int_{\mathbb{R}}|F(t)-F(t-u)| K_{n}(u) d u .
\end{aligned}
$$

Now $F$ is uniformly continuous on $\mathbb{R}$, as a continuous distribution function (this also follows from the stronger assumption (55)). By (50), the right hand side of (70) goes to zero as $n \rightarrow \infty$.

REMARK. From the expression (67) it is clear that $\zeta$ has the same distribution as $(B(F(t)), t \in \mathbb{R})$, where $B$ is the classical Brownian bridge indexed by $[0,1]$. This is useful to check the optimality of the Hölderian regularity order obtained in the conclusion of Theorem 16. To this end, consider the special distribution function

$$
F(t)= \begin{cases}0 & \text { if } t \leq 0 \\ t^{r} & \text { if } 0<t \leq 1 \\ 1 & \text { if } t>1\end{cases}
$$

which obviously satisfies (55). For the corresponding limiting process $\zeta$, we have

and

$$
\sup _{|t-s| \leq 1} \frac{|\zeta(t)-\zeta(s)|}{|t-s|^{r / 2}} \geq \limsup _{t \rightarrow 0^{+}} \frac{|\zeta(t)|}{t^{r / 2}}
$$

$$
\begin{aligned}
\limsup _{t \rightarrow 0^{+}} \frac{|\zeta(t)|}{t^{r / 2}} & =\limsup _{t \rightarrow 0^{+}} \frac{|B(F(t))|}{F(t)^{1 / 2}} \quad \text { (in distribution) } \\
& =\limsup _{u \rightarrow 0^{+}} \frac{W(u)}{u^{1 / 2}}=\infty \quad \text { a.s., }
\end{aligned}
$$


by a classical result of Lévy on the Brownian motion $W$ (see for instance [9]). Hence with probability one the paths of $\zeta$ do not belong to $C_{0}^{r / 2,0}$ and the conclusion of Theorem 16 cannot be improved.

\section{References}

[1] P. Baldi and B. Roynette, Some exact equivalents for the Brownian motion in Hölder norm, Probab. Theory Related Fields 93 (1993), 457-484.

[2] P. Billingsley, Convergence of Probability Measures, Wiley, 1968.

[3] Z. Ciesielski, On the isomorphisms of the spaces $H_{\alpha}$ and $m$, Bull. Acad. Polon. Sci. Sér. Sci. Math. Astronom. Phys. 8 (1960), 217-222.

[4] - Hölder conditions for realizations of Gaussian processes, Trans. Amer. Math. Soc. 99 (1961), 403-413.

[5] Z. Ciesielski, G. Kerkyacharian et B. Roynette, Quelques espaces fonctionnels associés à des processus gaussiens, Studia Math. 107 (1993), 171-204.

[6] L. T. Fernholz, Almost sure convergence of smoothed empirical distribution functions, Scand. J. Statist. 18 (1991), 255-262.

[7] D. Hamadouche, Invariance principles in Hölder spaces, Portugal. Math. (1998), to appear.

[8] —, Weak convergence of smoothed empirical process in Hölder spaces, Statist. Probab. Letters 36 (1998), 393-400.

[9] T. Hida, Brownian Motion, Springer, 1980.

[10] G. Kerkyacharian et B. Roynette, Une démonstration simple des théorèmes de Kolmogorov, Donsker et Ito-Nisio, C. R. Acad. Sci. Paris Sér. I 312 (1991), 877-882.

[11] J. Lamperti, On convergence of stochastic processes, Trans. Amer. Math. Soc. 104 (1962), 430-435.

[12] Yu. V. Prohorov [Yu. V. Prokhorov], Convergence of random processes and limit theorems in probability theory, Theory Probab. Appl. 1 (1956), 157-214.

[13] G. R. Shorack and J. A. Wellner, Empirical Processes with Applications to Statistics, Wiley, 1986.

[14] I. Singer, Bases in Banach Spaces II, Springer, 1981.

[15] S. Sun, Perturbed empirical distribution functions and quantiles under dependence, J. Theoret. Probab. 8 (1995), 763-777.

[16] C. Suquet, Tightness in Schauder decomposable Banach spaces, Translations of A.M.S., Proceedings of the St Petersburg Math. Soc., to appear.

Djamel Hamadouche and Charles Suquet

Laboratoire de Statistique et Probabilités, Bât. M2

Université des Sciences et Technologies de Lille

F-59655 Villeneuve d'Ascq Cedex, France

E-mail: Charles.Suquet@univ-lille1.fr 